\title{
ARTICLE \\ Curculigoside facilitates fear extinction and prevents depression-like behaviors in a mouse learned helplessness model through increasing hippocampal BDNF
}

\author{
San-juan Yang ${ }^{1}$, Zhu-jin Song ${ }^{1}$, Xun-cui Wang ${ }^{1}$, Zheng-rong Zhang ${ }^{1}$, Sheng-bing $\mathrm{Wu}^{1,2}$ and Guo-qi Zhu ${ }^{1,2}$
}

Curculigoside (CUR) is the main active component of traditional Chinese medicine Curculigoorchioides Gaertn (Xianmao in Chinese), which exhibits a variety of pharmacological activities. In this study we investigated the effects of CUR on fear extinction and related depression-like behaviors in mice. In fear conditioning task, we found that administration of CUR $\left(1.6,8,40 \mathrm{mg} \cdot \mathrm{kg}^{-1} \cdot \mathrm{d}^{-1}\right.$, ip, for 7 days) did not affect memory consolidation, but CUR at higher doses $\left(8,40 \mathrm{mg} \cdot \mathrm{kg}^{-1} \cdot \mathrm{d}^{-1}\right)$ significantly facilitated fear extinction, especially on D3 and D4. Moreover, CUR administration significantly ameliorated the fear conditioning-induced depression-like behaviors, likely through promoting fear extinction. We showed that CUR increased the expression of brain-derived neurotrophic factor (BDNF) and phosphorylation of tropomyosin receptor kinase B (TrkB) in the hippocampus, and activated protein kinase B (Akt)mammalian target of the rapamycin (mTOR) signaling pathway. Administration of the selective TrkB agonist 7,8-dihydroxyflavone $\left(7,8-\mathrm{DHF}, 5 \mathrm{mg} \cdot \mathrm{kg}^{-1} \cdot \mathrm{d}^{-1}\right.$, ip) also facilitated fear extinction, ameliorated depression-like behaviors. We established a mouse learned helplessness (LH) model to evaluate the antidepressant activity of CUR. The spatial memory was assessed in Morris water maze. We showed that LH-induced depression-like behaviors, including prolonged immobility times in forced swim and tail suspension tests as well as spatial memory impairments; LH also downregulated BDNF expression and the Akt-mTOR signaling pathway in the hippocampus. Administration of CUR $\left(1.6,8,40 \mathrm{mg} \cdot \mathrm{kg}^{-1} \cdot \mathrm{d}^{-1}\right.$, ip, for 14 days) or 7,8 -DHF $\left(5 \mathrm{mg} \cdot \mathrm{kg}^{-1} \cdot \mathrm{d}^{-1}\right.$, ip, for 3 days $)$ prevented LH-induced depression-like behaviors and promoted BDNF expression and the Akt-mTOR signaling pathway. In conclusion, CUR can accelerate the fear memory extinction and ameliorate depression-like behaviors in mice via promoting BDNF expression and activating the Akt-mTOR signaling pathway in the hippocampus.

Keywords: depression; curculigoside; 7; 8-dihydroxyflavone; fear extinction; learned helplessness model; BDNF; TrkB; Akt-mTOR signaling pathway; hippocampus

Acta Pharmacologica Sinica (2019) 40:1269-1278; https://doi.org/10.1038/s41401-019-0238-4

\section{INTRODUCTION}

Depression is a major global burden, affecting more than 300 million people worldwide [1, 2]. The features of this complex mental illness include persistent sadness or lack of pleasure with cognitive and physical symptoms (e.g., appetite loss, abnormal sleep patterns, low energy levels, decreased attention or physical activity, feelings of low value, and guilt) [3]. In 2002, the World Health Organization identified depression as the fourth most prevalent condition. In addition to its widespread incidence, depression is characterized by high rates of recurrence, disability, and mortality [4]. Approximately $30 \%$ of cases are refractory to pharmacologic treatment, and $70 \%$ of the patients do not recover fully [5].

Fear is a basic defensive emotion of human beings that is of great significance in the survival and adaptation of individuals. However, a lack of effective fear suppression can lead to overexpression of fear and the occurrence of corresponding psychological disorders, such as depression, panic disorder, and posttraumatic stress disorder (PTSD) $[6,7]$. PTSD is a type of abnormal mental reaction after severe traumatic stress, such as war, natural disasters, serious accidents, and violent crimes. PTSD is characterized by fear memory, especially loss of damaged memory or overrecall of fear events. PTSD is often accompanied by other mental disorders, such as depression and anxiety disorders. Exposure therapy refers to the process of recollecting the trauma to allow patients to become accustomed to the fear and eliminate it. A clinical study indicated that exposure therapy is an effective treatment for PTSD [8, 9]. Like most psychiatric interventions, exposure-based psychotherapy has some drawbacks and has no obvious effect in some patients [10]. The core reason for its ineffectiveness is an inability for the fear memory to be extinguished or at least not completely extinguished after exposure therapy. In addition to exposure therapy, drugassisted therapy is also needed to accelerate the extinction of fear memory [11].

Rhizoma Curculiginis (Xianmao in Chinese) is a commonly used drug in traditional Chinese medicine; its functions include tonifying kidney yang and improving blood supply [12, 13].

\footnotetext{
${ }^{1}$ Key Laboratory of Xin'an Medicine, Ministry of Education, Anhui University of Chinese Medicine, Hefei 230038, China and ${ }^{2}$ Anhui Academy of Chinese Medicine, Hefei 230038, China

Correspondence: Guo-qi Zhu (guoqizhu@gmail.com)
}

Received: 9 January 2019 Accepted: 11 April 2019

Published online: 26 April 2019 
Xianmao is one of the main ingredients of several prescriptions used to treat depression symptoms, such as the "Er-xian Decoction" [14]. Curculigoside (CUR, $\mathrm{C}_{22} \mathrm{H}_{26} \mathrm{O}_{11}$ ) is the main active component of Rhizoma Curculiginis. CUR has a phenolic structure, can pass the blood-brain barrier and is widely distributed in the brain for at least $4 \mathrm{~h}$ after oral ingestion [15]. The pharmacological activities of CUR have been extensively investigated, including its antioxidant activities [16], cardiovascular protection [17], immune regulation [18], and neuroprotective effects [19]. However, how CUR affects fear and fear-related depression-like symptoms is unclear.

Brain-derived neurotrophic factor (BDNF) is one of the most important members of the neurotrophic factor family and is essential for cell survival and synaptic plasticity [20, 21]. BDNF levels are reduced in patients with chronic depression who have experienced chronic stress events [22]. Other studies have reported lower levels of BDNF in the hippocampus and prefrontal cortex of suicidal depression patients, with increased expression in patients treated with antidepressant drugs [23, 24]. Because depression is closely related to patients' fear of traumatic memories [25], we employed a learned helplessness (LH) mouse model to investigate the protective effects of CUR and the associated mechanisms.

\section{MATERIALS AND METHODS}

Animals and drugs

Male C57BL/6 mice (3 months old; body weight: $20-25 \mathrm{~g}$ ) were purchased from the Experimental Animal Center of Anhui Medical University [SCXK (Anhui) 2011-002]. The protocol was approved by the Ethics Committee of Anhui University of Chinese Medicine. Mice were housed in a temperature-controlled room with a standard $12 \mathrm{~h}$ light $/ 12 \mathrm{~h}$ dark cycle and access to food and water ad libitum. The animals were adapted for 1 week prior to experimentation.

CUR was obtained from BIOX (HPLC $\geq 98.94 \%$, BIOX, USA). 7,8-dihydroxyflavone (7,8-DHF) was purchased from Selleckchem (HPLC $=99.01 \%$, Selleckchem, USA). After CUR was dissolved in DMSO, it was suspended in normal saline (DMSO concentration was lower than $0.1 \%$ ), and the control group was injected with the same volume of normal saline (containing the same amount of DMSO).
Groups and drug administration

Experimental protocol 1: fear conditioning task. Fear memory and extinction were produced as previously described [26]. The fear conditioning box (UgoBasile, Gemonio, Italy) contained a camera that was connected to ANY-Maze software (Stoelting Co., Wood Dale, IL, USA). After CUR administration $(1.6-40 \mathrm{mg} / \mathrm{kg}$ ) for 7 continuous days (once per day) (Fig. 1), the animals underwent a fear memory and extinction protocol. The doses of CUR were selected based upon a previous publication [27]. The mice were placed in the fear conditioning box for $5 \mathrm{~min}$ and exposed to three electric foot shocks $(1.0 \mathrm{~mA}, 2 \mathrm{~s}$, and $73-74 \mathrm{~s}$ in interval). Twenty-four hours later, the mice were returned to the fear conditioning box and exposed to the fear context for $15 \mathrm{~min}$ without electric shock. The amount of time each mouse spent freezing in the $15 \mathrm{~min}$ was recorded, and freezing time in the initial 5 min was statistically analyzed. The mice were put into the fear conditioning box for 5 min at the same time every day for 3 extinction days. The conditioning chamber was cleaned with $70 \%$ ethanol to remove background odor. One hour after extinction, a forced swimming test (FST) and a tail suspension test (TST) were conducted. In the fear memory experiment, mice in the 7,8-DHF group were intraperitoneally injected with 7,8-DHF (5 mg/kg) $30 \mathrm{~min}$ before re-exposure (Fig. 1).

Experimental protocol 2: learned helplessness model. Depressionlike behaviors were induced by the LH paradigm as described in a previous study [28]. The LH paradigm was performed with the use of the fear conditioning box (UgoBasile, Gemonio, Italy), which was controlled by ANY-Maze software (Stoelting Co., Wood Dale, $\mathrm{IL}$, USA). On the 11 th day after drug administration, mice in the $\mathrm{LH}$, $\mathrm{LH}+\mathrm{DMSO}$, and $\mathrm{LH}+\mathrm{CUR}(40,8$, and $1.6 \mathrm{mg} / \mathrm{kg})$ groups were subjected to 30 inescapable electric foot shocks $(0.70 \mathrm{~mA}, 20 \mathrm{~s}$ duration, random intervals) in the same compartment for 3 consecutive days. Control mice were placed in the same environment but did not receive foot shocks. To prevent odor interference, the boxes were cleaned with $75 \%$ alcohol between sessions. The CUR treatment groups received daily intraperitoneal injections of 40,8 , or $1.6 \mathrm{mg} / \mathrm{kg}$ CUR for 14 consecutive days, as shown in Fig. 1. The LH mice in the 7,8-DHF group received three injections of $7,8-\mathrm{DHF}(5 \mathrm{mg} / \mathrm{kg}) 30 \mathrm{~min}$ before the daily electric shock sessions. Behavioral experiments were performed $24 \mathrm{~h}$ after the end of the electric shock (Fig. 1).
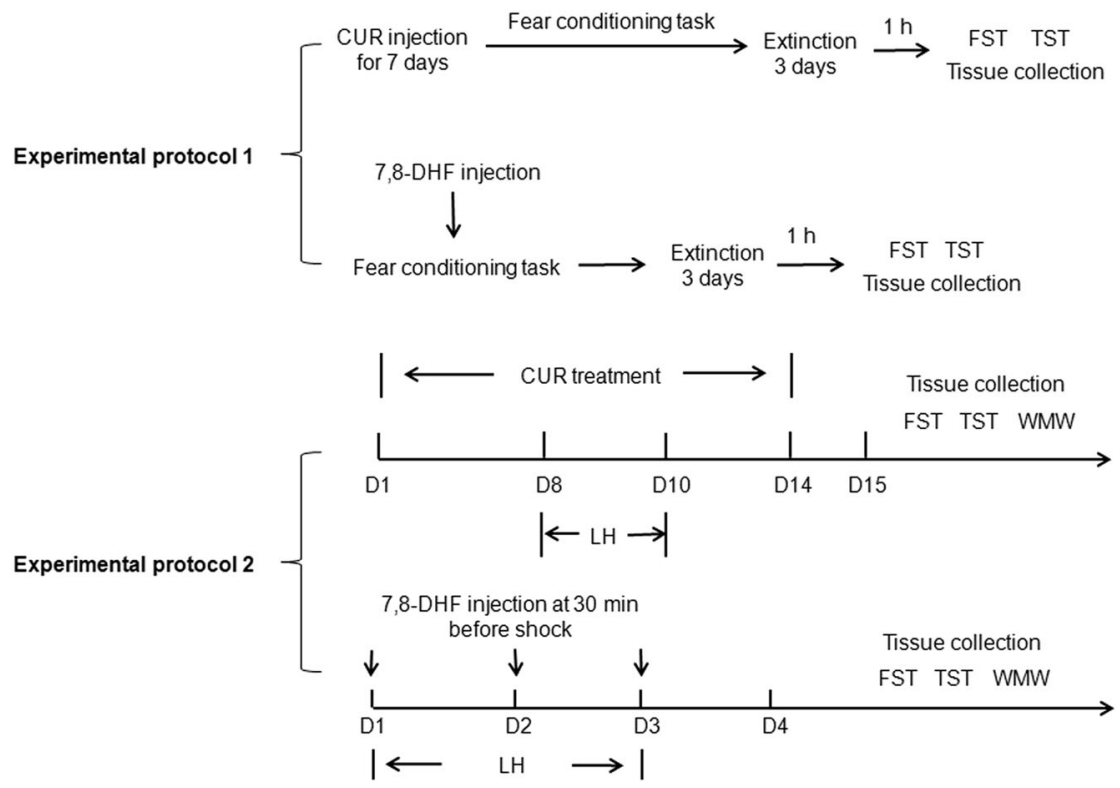

Fig. 1 Schematics illustrate the study designs. Upper panel: experimental protocol 1; Down panel: experimental protocol 2 
Behavioral tests

Tail suspension test (TST). The TST protocol was similar to that described by Steru et al. [29]. After 14 days of CUR administration, each mouse was suspended on the edge of a shelf $\sim 50 \mathrm{~cm}$ above the bottom of the sound insulation box using adhesive tape placed $\sim 1 \mathrm{~cm}$ from the tip of the tail. The animals were allowed to move for $6 \mathrm{~min}$, and behavior was recorded with SuperMaze software (Shanghai Xinruan Information Technology Co. Ltd., Shanghai, China). The duration of immobility was recorded in the last $4 \mathrm{~min}$.

Forced swimming test (FST). After the TST, the mice were allowed to rest for $24 \mathrm{~h}$. The mice were then individually placed in a $2000 \mathrm{~mL}$ glass container with water temperature at $22 \pm 3^{\circ} \mathrm{C}$. Super Maze software was used to record the movement. The duration of immobility was recorded during the last 4 min of the test.

Morris water maze (MWM). The MWM was used to evaluate learning and memory in the mice undergoing experimental protocol 2. Mice were allowed to rest for $24 \mathrm{~h}$ after the FST. Then, the MWM test was performed as described previously [30, 31]. Briefly, the test was conducted in a pool $(120 \mathrm{~cm}$ diameter) filled with water $\left(23 \pm 2{ }^{\circ} \mathrm{C}\right)$. The pool was imaginarily divided into four quadrants. The test comprised two trials: a 4-day spatial navigation trial (learning) and a 1 day probe trial (memory). In the spatial navigation trial, an escape platform, always located in the first (target) quadrant, was submerged $1 \mathrm{~cm}$ beneath the water surface. In each spatial navigation trial, the time to reach platform from the starting location was recorded as latency. Each trial lasted a maximum of $60 \mathrm{~s}$. If the mouse failed to find the platform within $60 \mathrm{~s}$, it was manually guided to the platform and allowed to stay for $15 \mathrm{~s}$, and the latency was recorded as $60 \mathrm{~s}$. After the last spatial navigation trial, all mice completed the probe trial, in which the platform was removed, to assess the final strength of the memory trace. In probe trials, times spent in the platform region were recorded. The mice were placed in the contralateral quadrant, and the time spent in the target quadrant was recorded and calculated with Ugo Basile software.

Immunochemical staining. The hippocampi were isolated and kept at $-80^{\circ} \mathrm{C}$ or fixed in $4 \%$ paraformaldehyde. After fixation in $4 \%$ paraformaldehyde for $24 \mathrm{~h}$, brain tissue was cryoprotected in $30 \%$ sucrose for $24 \mathrm{~h}$ at $4{ }^{\circ} \mathrm{C}$ and sectioned on a freezing microtome at $20 \mu \mathrm{m}$. Sections were blocked in $0.1 \mathrm{M}$ phosphatebuffered saline (PBS) containing $10 \%$ goat serum and $0.4 \%$ Triton $\mathrm{X}-100$ for $1 \mathrm{~h}$ and then incubated with a primary antibody against p-mTOR (1:200, Santa Cruz Biotechnology, Santa Cruz, CA, USA) in $0.1 \mathrm{M}$ PBS containing $5 \%$ goat serum and $0.4 \%$ Triton $\mathrm{X}-100$ overnight at $4{ }^{\circ} \mathrm{C}$. Sections were washed three times ( $15 \mathrm{~min}$ each) in PBS and incubated with fluorescein (FITC)-conjugated goat antirabbit lgG $(\mathrm{H}+\mathrm{L})$ (Life Technologies, Carlsbad, CA, USA) for $2 \mathrm{~h}$ at room temperature. Images were taken with an FV1000 Olympus confocal laser scanning microscope (Olympus, Tokyo, Japan).

Western blotting. Hippocampal homogenates from animals were obtained from each group and lysed. Bicinchoninic Acid Kits (Beyotime Institute of Biotechnology) were used to detect protein concentrations. Protein samples were separated by sodium dodecyl sulfate polyacrylamide gel electrophoresis ( $12 \%$ gels) for $50 \mathrm{~min}$ at $120 \mathrm{~V}$ and transferred onto a nitrocellulose membrane for $2 \mathrm{~h}$ at $200 \mathrm{~mA}$. The membranes were blocked with PBS containing $0.1 \%$ Tween-20 (PBST) and 5\% fat-free milk for $2 \mathrm{~h}$ at room temperature and then incubated with rabbit antibodies against p-Akt (1:1000, Cell Signaling Technology [CST], Danvers, MA, USA), Akt (1:1000, CST), p-mTOR (1:500, Santa Cruz Biotechnology, Santa Cruz, CA, USA), mTOR (1:500, Santa Cruz Biotechnology), BDNF (1:1000, CST), PSD95 (1:1000, CST), p-ERK
(1:1000, CST), ERK (1:1000, CST), phospho-TrkB (1:3000, Cell Signaling, Danvers, MA, USA), TrkB (1:3000, Cell Signaling), glyceraldehyde 3-phosphate dehydrogenase (GAPDH), rabbit IgG (1:1000, Zs-bio, Beijing, China), or $\beta$-actin (1:1000, Zs-bio) overnight at $4{ }^{\circ} \mathrm{C}$, followed by a 2 -h incubation with a peroxidaseconjugated goat anti-rabbit IgG $(1: 10,000$, Zs-bio) at room temperature. Band intensity was densitometrically quantified using ImageJ software. Quantification of total protein was determined relative to $\beta$-actin, whereas phospho-protein level was determined relative to total protein. Ratios of total protein/ actin or phospho-protein/total protein in experimental groups were obtained for each experiment and normalized to control values to avoid within-group variations.

\section{ELISA}

The hippocampus tissue was weighed. An appropriate volume of PBS $(1 \mathrm{~mL} / 10 \mathrm{mg}$ ) by weight was added to a portion of the hippocampal tissue, which was then rapidly homogenized, and the cells were disrupted by an ultrasonic cell disrupter (Scietz Biotechnology Co. Ltd, Ningbo, China). After the samples were centrifuged $(5870 \times g$ for $5 \mathrm{~min}$ ), the supernatant was collected, and protein quantification was performed. The ELISA was performed according to the instructions of the kit (Beyotime Institute of Biotechnology, China). The absorbance value was quickly read on the microplate reader at a detection wavelength of $450 \mathrm{~nm}$.

\section{Statistical analysis}

Data were analyzed with GraphPad Prism 5.0 software (GraphPad Inc., San Diego, CA, USA) and are presented as the means \pm SEM; $P<0.05$ was considered significant. Student's $t$ test or one-way ANOVA followed by Bonferroni testing was applied to compare between-group differences.

\section{RESULTS}

CUR facilitates fear memory extinction

Freezing during the initial $5 \mathrm{~min}$ of re-exposure was used to indicate the fear memory of the mice. Our results showed that there was no significant difference in freezing among groups (Fig. 2a, $F_{(3,30)}=0.7721, P>0.05$ ), suggesting that CUR treatment did not affect the formation of fear memory. Figure $2 b$ shows the extinction data of the mice in each group. With increasing time, freezing time decreased in each group. On the 4th day, the mice in the CUR $(40 \mathrm{mg} / \mathrm{kg})$ and CUR $(8 \mathrm{mg} / \mathrm{kg})$ groups had significantly lower freezing times than those in the model group $(P<0.05)$. These results suggest that CUR administration facilitated fear memory extinction in mice.

\section{CUR prevents depression-like activity in animals receiving fear} extinction

After fear extinction, the mice underwent forced swimming and tail suspension tests. Our data showed that the immobility times of FM mice in the FST $\left(F_{(5,35)}=52.85\right)$ and TST $\left(F_{(5,35)}=11.66\right)$ were significantly greater than those of control mice (who did not experience the fear memory extinction protocol) (Fig. 3a, b, $P<$ $0.05)$. In contrast, compared with the immobility times of mice in the FM group, the immobility times of mice treated with different doses of CUR were significantly lower $(P<0.05)$. The data indicated that FM mice displayed depression-like behaviors and that CUR treatment improved these depression-like behaviors. We also analyzed the correlation between the freezing time on the last day and the immobility times in the FST and TST. The results showed that freezing time was positively correlated with immobility times in the TST (Fig. 3c, $r=0.4321$ ) and FST (Fig. 3d, $r=0.6351$ ). These data indicated that fear memory was positively correlated with depressive behavior and that CUR might exert its effect on depression-like behaviors by facilitating fear extinction. 

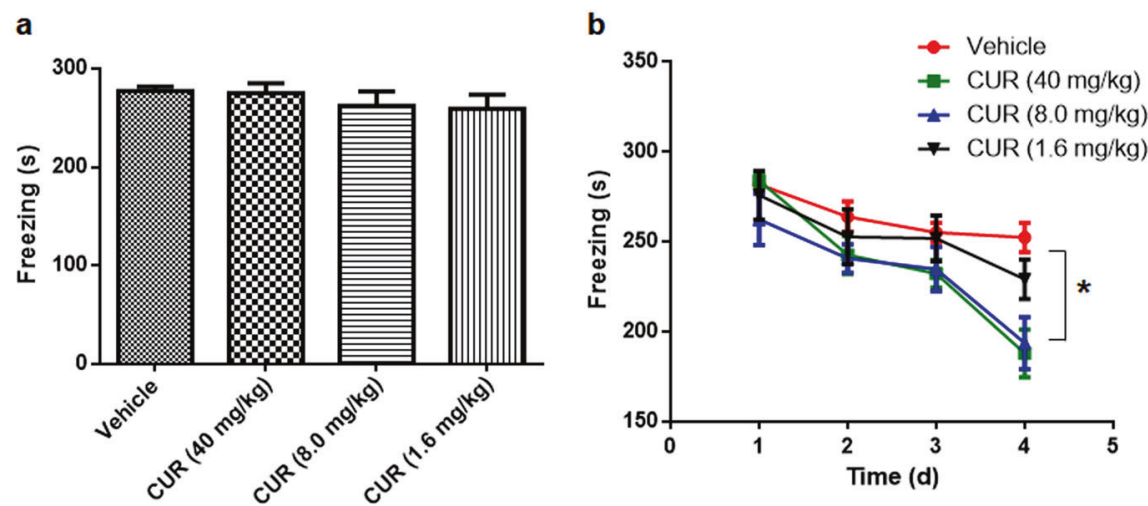

Fig. 2 Curculigoside (CUR) facilitates fear memory extinction. a The freezing time in the first 5 min of re-exposure. $\mathbf{b}$ The extinction of fear memory. ${ }^{*} P<0.05$ versus vehicle

a
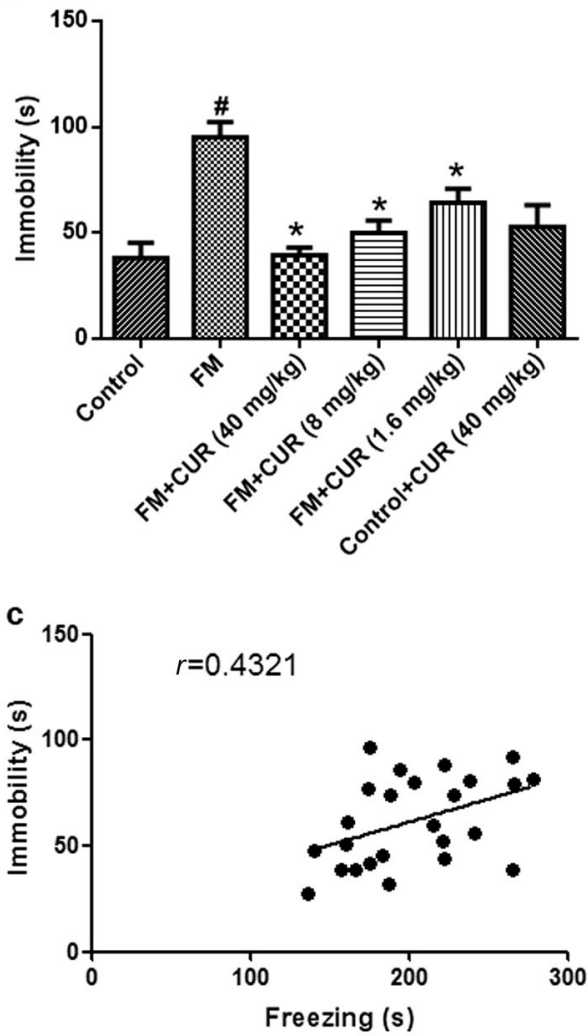

b

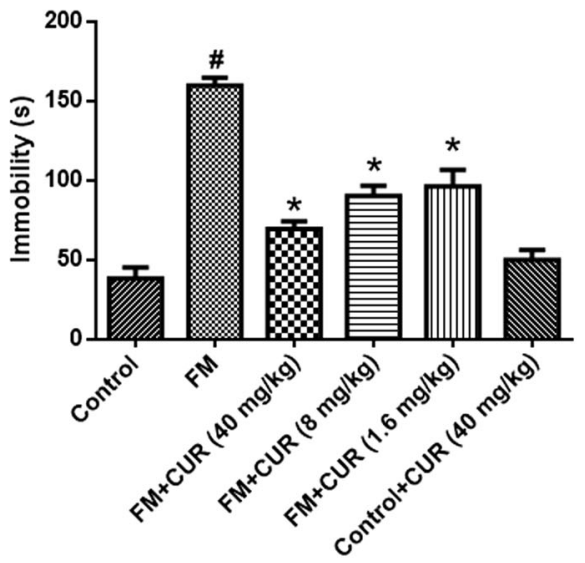

d

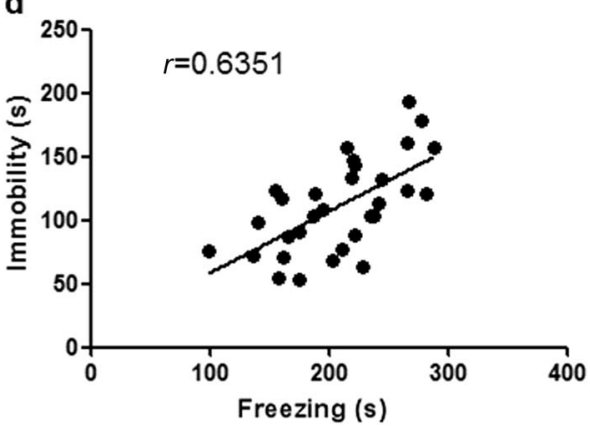

Fig. 3 Curculigoside (CUR) prevents depression-like activity in animals receiving fear extinction. a Immobility time in the tail suspension test. b Immobility time in the forced swim test. c Analysis of the correlation between the immobility time in the tail suspension test and the freezing time. $\mathbf{d}$ Analysis of the correlation between the immobility time in the forced swim test and the freezing time. ${ }^{\#} P<0.05$ versus control, ${ }^{*} P<0.05$ versus FM. FM represents the procedure of fear conditioning task, including memory formation, consolidation, and extinction

CUR prevents fear extinction and depression-like behaviors by increasing BDNF

BDNF is associated with fear memory extinction [32, 33]. We detected the expression of BDNF in the hippocampus. The expression of BDNF in FM mice was significantly lower in those treated with CUR (Fig. $4 \mathrm{a}, F_{(3,10)}=4.270$, versus FM, $P<0.05$ ). Meanwhile, the expression of BDNF in the hippocampus of normal mice was also significantly lower in those given CUR (Fig. 4b, $t=$ 3.936, versus control, $P<0.05$ ). These results suggest that CUR not only increases the expression of BDNF in the hippocampus of normal mice but also increases the expression of BDNF in FM mice.

7,8-DHF has been shown to increase the expression of BDNF $[34,35]$. To further confirm that the effect of CUR on fear extinction and depression was through increasing BDNF levels, we treated mice with 7,8-DHF. Our results showed that 7,8-DHF significantly increased BDNF levels in FM mice (Fig. 4c, $t=7.456$, $d f=4, \quad P<0.05)$. ELISA also showed that CUR and 7,8-DHF promoted BDNF levels in control and FM mice (Fig. $4 d, F_{(4,15)}=$ 14.59). Moreover, the $\mathrm{p}$-TrkB level in the FM group was lower than 
a
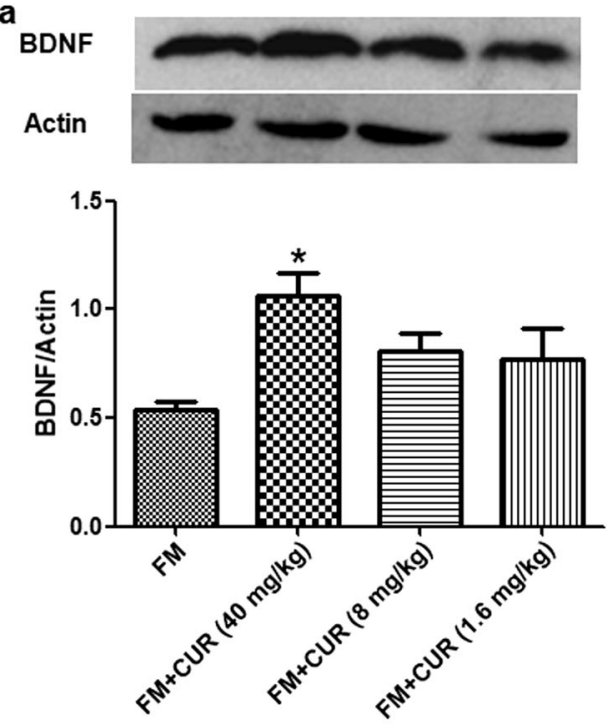

d

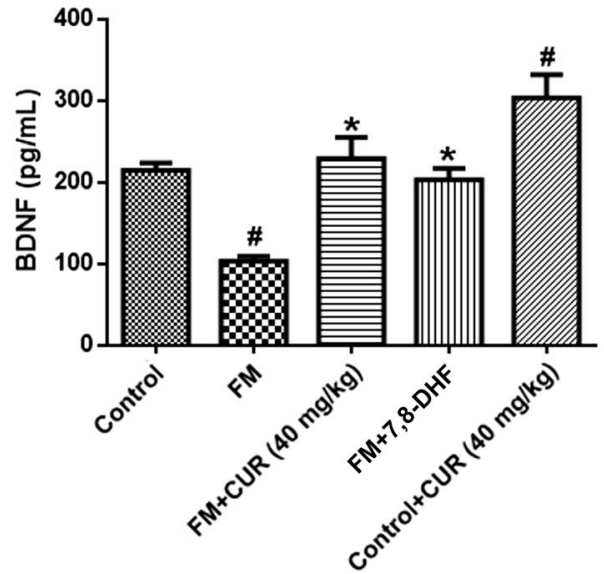

f

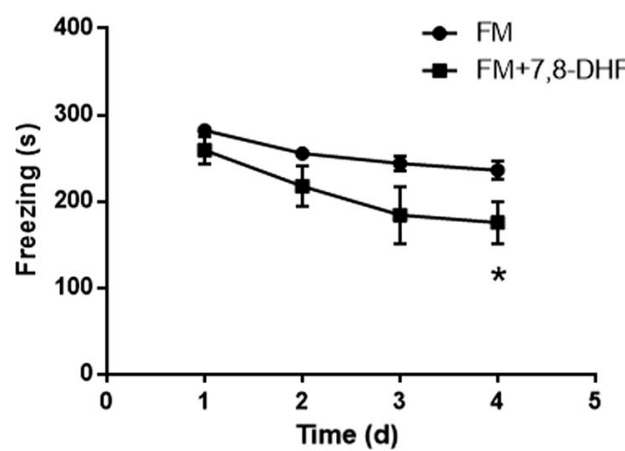

b
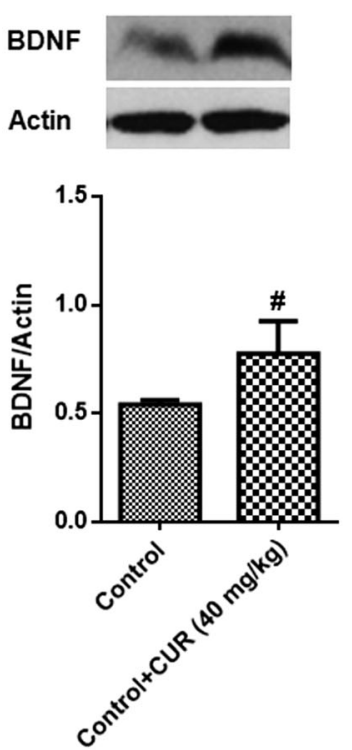

C
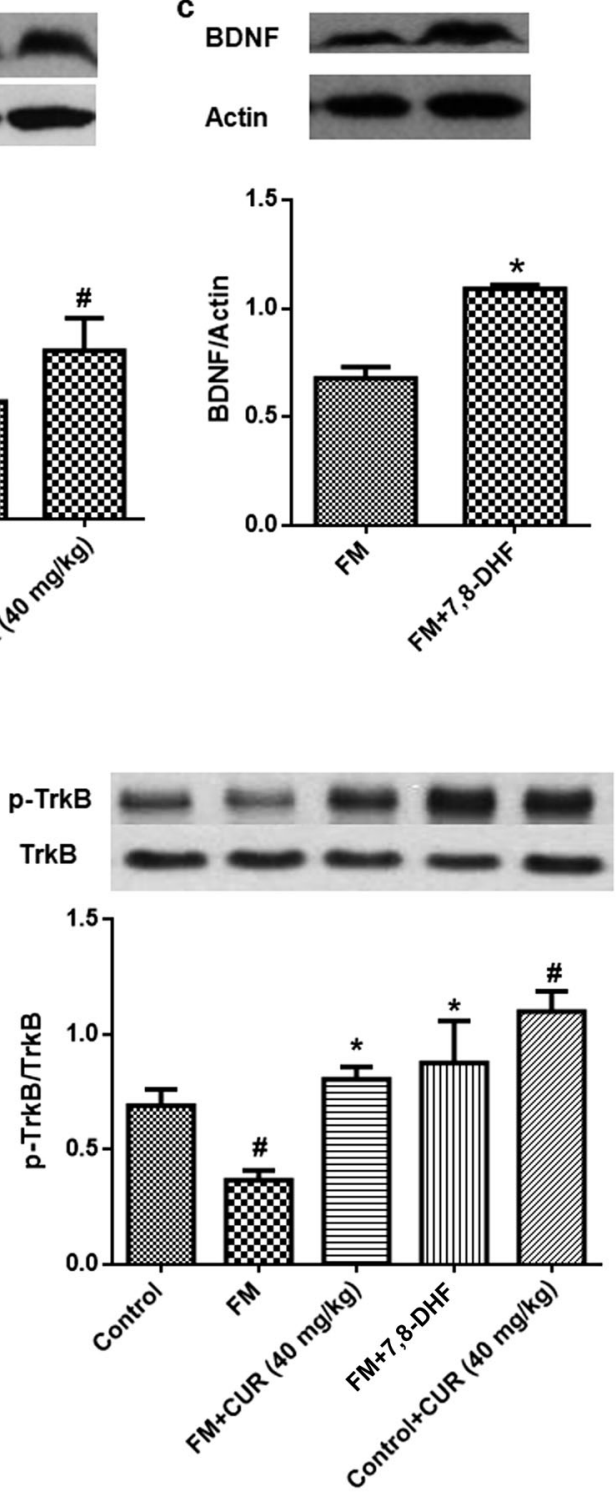

g

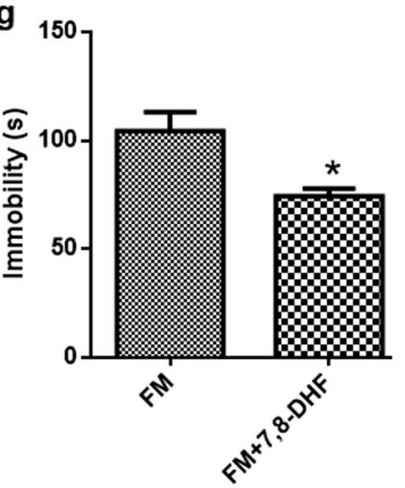

h

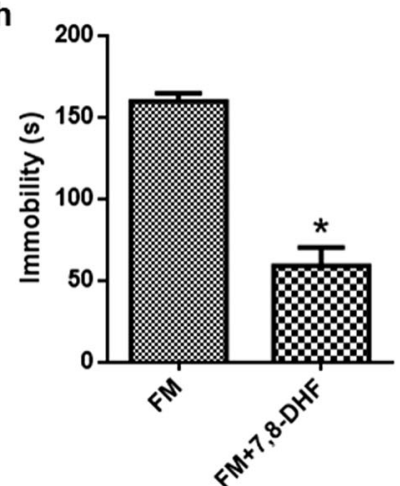

Fig. 4 Curculigoside (CUR) prevents fear memory extinction and depression-like activity through increasing BDNF and activating TrkB phosphorylation. a Upper panels are representative blots of BDNF and actin; the lower panel shows BDNF/actin quantification data in CUR treatment. $\mathbf{b}$ Upper panels are representative blots of BDNF and actin; the lower panel shows BDNF/actin quantification data in control mice. c Upper panels are representative blots of BDNF and actin; the lower panel shows BDNF/actin quantification data; $\mathbf{d}$ ELISA detecting BDNF level; e Upper panels are representative blots of $p$-TrkB and TrkB; the lower panel shows $\mathrm{p}$-TrkB/TrkB quantification data; $\mathbf{f}$ 7,8-DHF treatment facilitates memory extinction; $\mathbf{g}$ Immobility time in the tail suspension test. $\mathbf{h}$ Immobility time in the forced swim test. ${ }^{\#} P<0.05$ versus control, ${ }^{*} P<0.05$ versus FM. FM represents the procedure of fear conditioning task, including memory formation, consolidation, and extinction 
that in the control group. CUR and 7,8-DHF promoted TrkB phosphorylation in control and FM mice (Fig. 4e).

On the fourth day of extinction, the freezing time in the 7,8-DHF-treated mice was significantly lower than that in the FM mice (Fig. 4f, $P<0.05$ ). Compared with the immobility times of the mice in the FM group, the immobility times of mice treated with $7,8-\mathrm{DHF}$ in the FST $(t=9.553, d f=13)$ and TST $(t=3.114, d f=8)$ were significantly lower (Fig. $4 \mathrm{~g}, \mathrm{~h}, P<0.05$ ). The above data indicate that, similar to 7,8-DHF, CUR increased BDNF levels to promote fear extinction and improve depression-like behaviors.

CUR activates the AKT-mTOR pathway

To investigate the signaling pathway mediated by CUR, we also detected the expression of the Akt-mTOR signaling pathway, which is related to protein synthesis [26]. The results showed that the expression of p-Akt and p-mTOR in the hippocampus of the CUR (40 mg/kg) group was significantly higher than that in the hippocampus of the FM group (Fig. 5a-c, $F_{(3,8)}=107.9, F_{(3,8)}=$ $7.721, P<0.05)$. However, there was no significant difference in the expression levels of PSD95 $(t=0.5419, d f=4)$ or p-ERK $(t=0.6507, d f=4)$ in the hippocampus (Fig. $5 d-f, P>0.05)$. After treatment with 7,8-DHF, the levels of p-Akt $(t=9.251, d f=4)$ and p-mTOR $(t=3.512, d f=4)$ in the hippocampus were also significantly higher than those in the FM group (Fig. $5 g-i$, $P<0.05)$. The immunofluorescence results of $p$-mTOR were consistent with those of Western blot (Fig. 5j). These results suggest that CUR might increase the expression of BDNF by enhancing the Akt-mTOR signaling pathway.

CUR ameliorates LH-induced depression-like activities and memory impairment

As CUR promotes fear memory extinction and related depression, we further verified whether CUR is able to improve depression-like behaviors in an LH model. The FST and TST were applied to assess
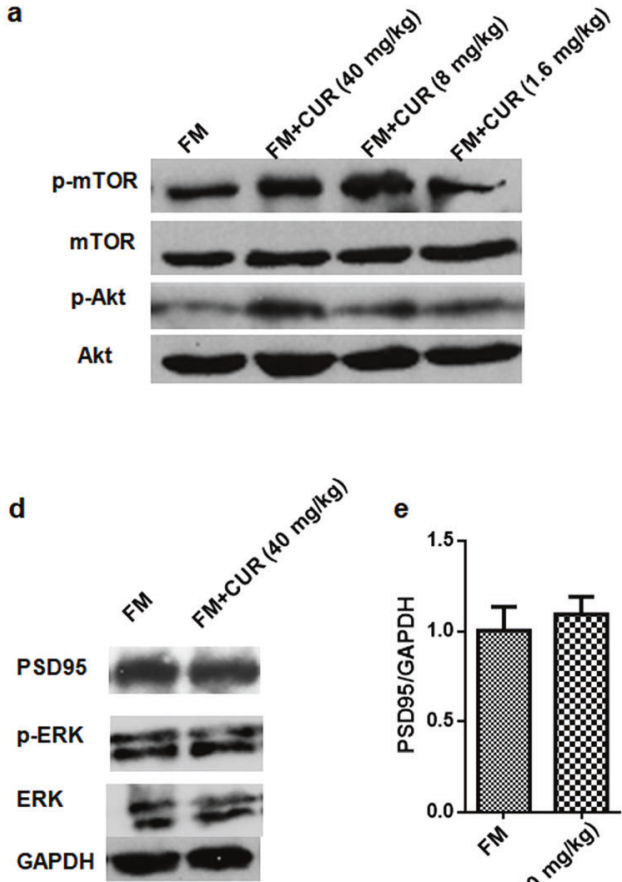

i

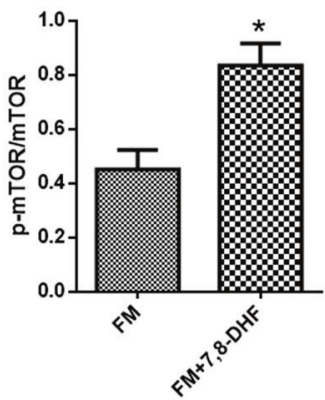

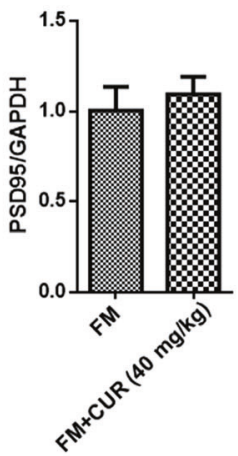

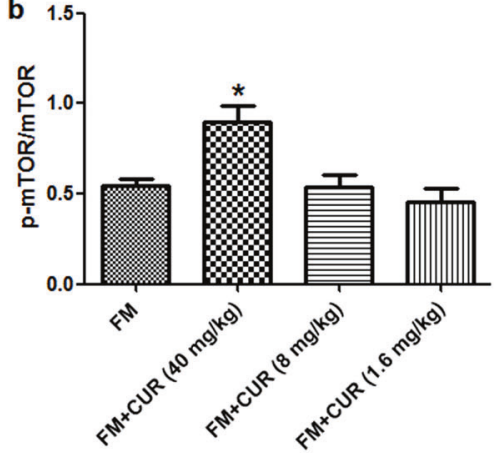

f

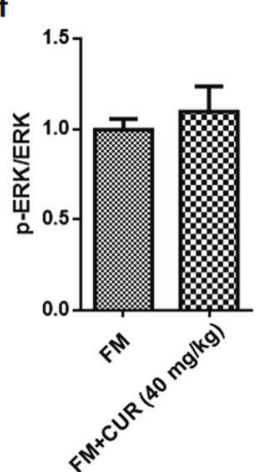

g
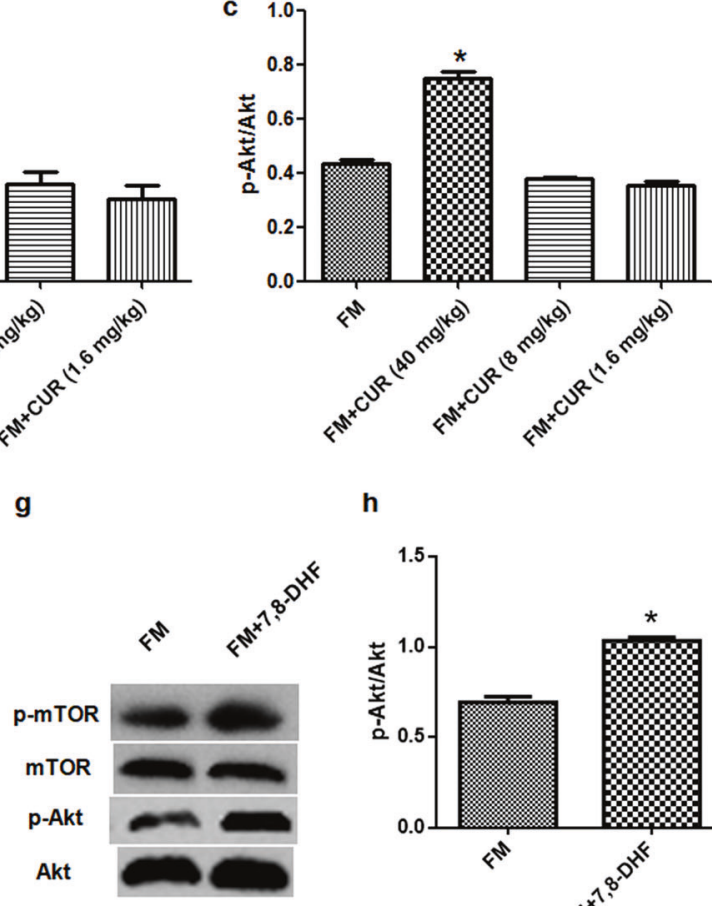

h

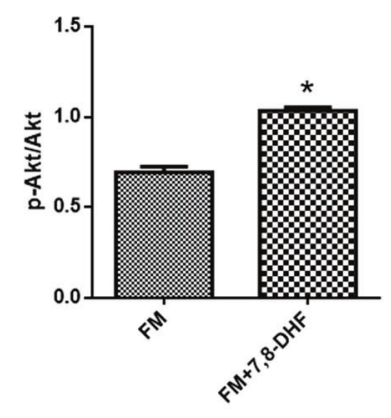

j

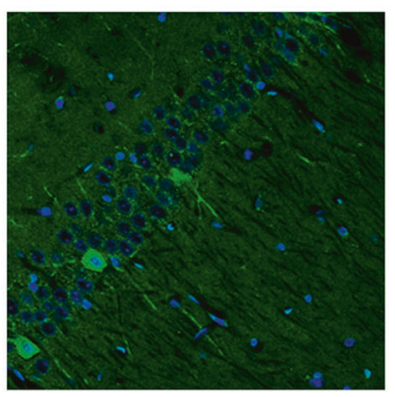

FM

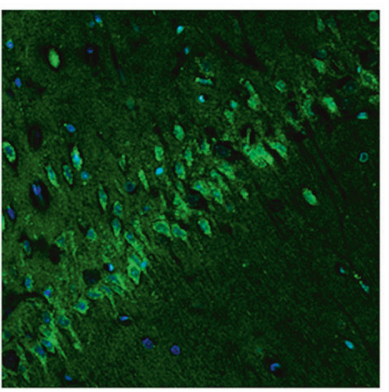

FM+CUR (40 mg/kg)

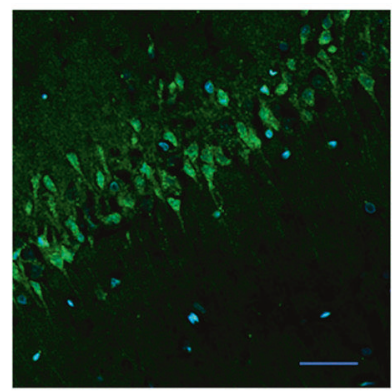

$\mathrm{FM}+7,8-\mathrm{DHF}$

Fig. 5 Curculigoside (CUR) activates protein kinase $B$ (p-AKT)-mammalian target of the rapamycin (mTOR) pathway through increasing BDNF. a Representative blots of p-mTOR, mTOR, p-Akt, and Akt. b p-mTOR/mTOR quantification data; c p-Akt/Akt quantification data. d Representative blots of PSD95, p-ERK, ERK, and GAPDH. e PSD95/GAPDH quantification data. f p-ERK/ERK quantification data. g Representative blots of p-mTOR, mTOR, p-Akt, and Akt after 7,8-DHF treatment. h p-Akt/Akt quantification data in 7,8-DHF treatment. i p-mTOR/mTOR quantification data in 7,8-DHF treatment. ${ }^{*} P<0.05$ versus FM; $\mathbf{j}$ Representative images of p-mTOR expression in hippocampal CA1 area. Scale bar: $100 \mu \mathrm{m}$. FM represents the procedure of fear conditioning task, including memory formation, consolidation, and extinction 
a
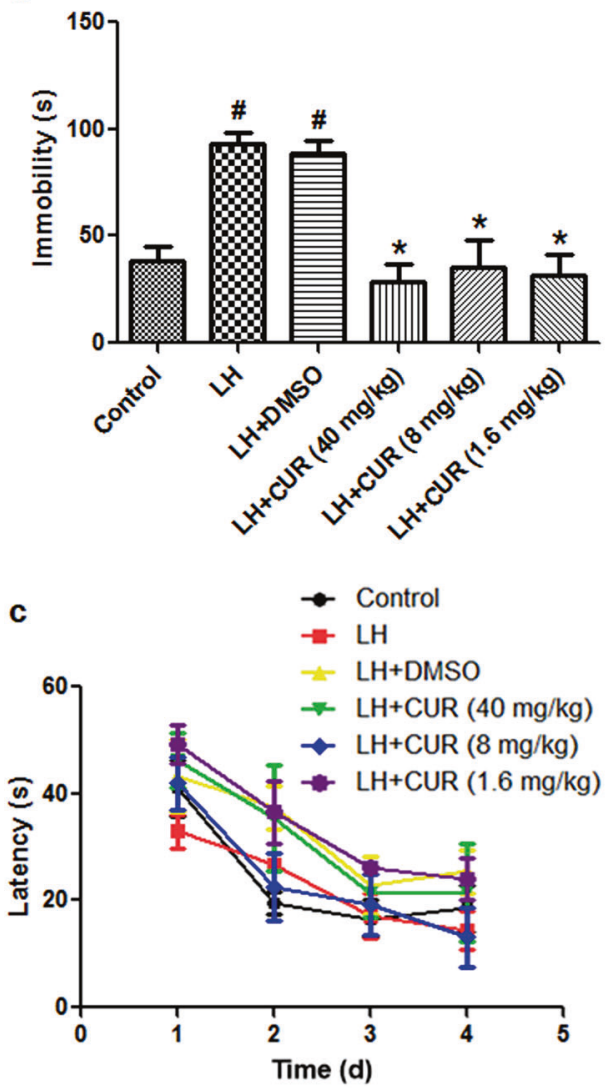

b
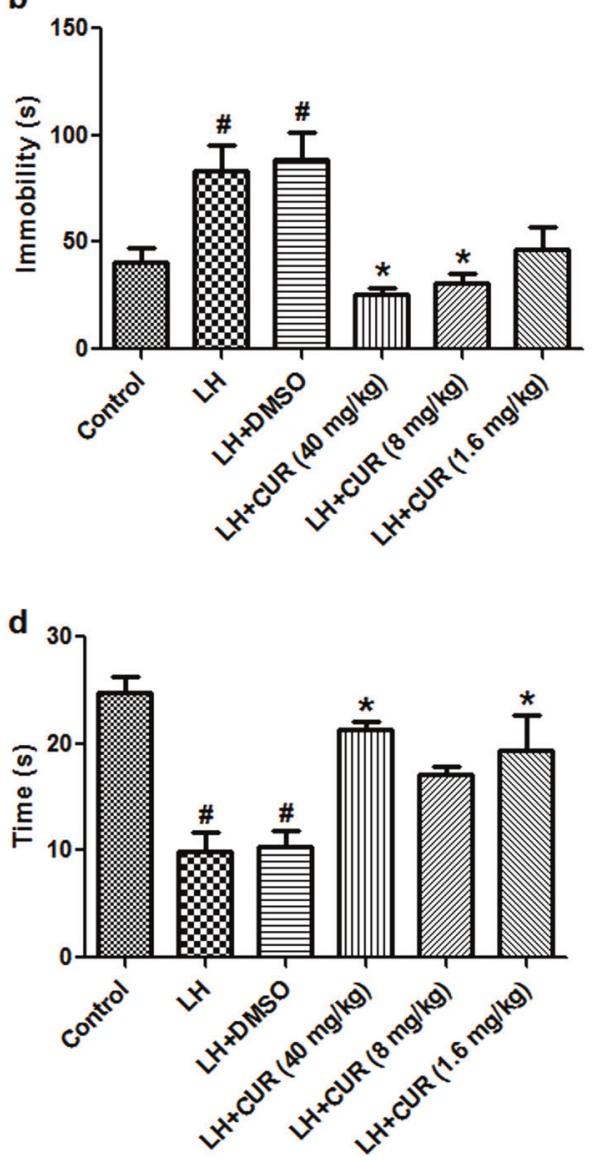

Fig. 6 Curculigoside (CUR) ameliorates learned helplessness (LH)-induced depression-like activities and memory impairment. a Immobility time in the tail suspension test. b Immobility time in the forced swim test. c Four-day learning session. $\mathbf{d}$ Time spent in target quadrant. ${ }^{\#} P<0.05$ versus control, ${ }^{*} P<0.05$ versus $\mathrm{LH}$

depression-like activity. In the TST, the immobility times in LH and $\mathrm{LH}+\mathrm{DMSO}$ mice were significantly higher than those in control mice $(P<0.05)$ (Fig. 6a, $\left.F_{(5,36)}=10.44\right)$, and these immobility times were reduced in the $\mathrm{LH}+\mathrm{CUR}(40,8$, and $1.6 \mathrm{mg} / \mathrm{kg})$ groups $(P<$ 0.05 for each comparison). As shown in Fig. $6 \mathrm{~b}$, the FST immobility times in the $\mathrm{LH}$ and $\mathrm{LH}+$ DMSO groups were significantly higher than those in the control group $\left(P<0.05, F_{(5,36)}=9.955\right)$, and these immobility times were reduced in the 40 and $8 \mathrm{mg} / \mathrm{kg} \mathrm{LH}+$ CUR groups ( $P<0.05$ versus model for each comparison). These data suggest that depression-like activity is induced in the $\mathrm{LH}$ model and that CUR can reduce depression-like behaviors in $\mathrm{LH}$ mice.

MWM testing was performed to assess the effects of CUR on learning and memory in LH mice. The latency to find the platform was prolonged in the LH group, but the difference was insignificant (Fig. 6c). After platform removal, the times spent in the target quadrant were significantly shorter in $\mathrm{LH}$ and $\mathrm{LH}+$ DMSO animals than in control animals (both $P<0.05$ versus control, $\left.F_{(5,36)}=10.85\right)$, but this effect was reversed in the 40 and $1.6 \mathrm{mg} / \mathrm{kg} \mathrm{LH}+$ CUR groups (both $P<0.05$ versus model, Fig. $6 \mathrm{~d}$ ). These data suggest that memory retrieval was impaired in LH mice but was ameliorated by CUR.

CUR increases hippocampal BDNF levels and Akt-mTOR signaling in the $\mathrm{LH}$ model

We also examined hippocampal BDNF levels. The results showed that BDNF expression was significantly lower in the $\mathrm{LH}$ and $\mathrm{LH}+$ DMSO groups than in the control group $(P<0.05$,
Fig. 7a, $\left.F_{(5,12)}=10.12\right)$. However, BDNF levels in the LH + CUR groups $(40,8$, and $1.6 \mathrm{mg} / \mathrm{kg})$ were higher than those in the LH group $(P<0.05, P<0.05$, and $P<0.05$, respectively). These data suggest that LH downregulated hippocampal BDNF levels, while CUR administration normalized the BDNF expression in LH mice.

We detected p-Akt and p-mTOR after CUR administration. As shown in Fig. 7b, Akt phosphorylation was significantly reduced in the $\mathrm{LH}$ and $\mathrm{LH}+$ DMSO groups (both $P<0.05$ versus control, $F_{(5}$, ${ }_{12}=19.76$ ). However, CUR ( 40 and $8 \mathrm{mg} / \mathrm{kg}$ ) treatment reversed the decrease in p-Akt $(P<0.05$ versus the LH group). The expression of p-mTOR $\left(F_{(5,12)}=9.054\right)$ in the LH and LH + DMSO groups was downregulated compared with that in the control group (both $P<0.05)$. However, CUR treatment $(40,8$, and $1.6 \mathrm{mg} /$ $\mathrm{kg})$ significantly increased $\mathrm{p}$-mTOR expression $(P<0.05$ versus the $\mathrm{LH}$ group, Fig. 7c). These results indicate that $\mathrm{LH}$ specifically attenuated Akt-mTOR signaling, and this attenuation was reversed by CUR.

7,8-DHF ameliorates depression-like behaviors in LH mice and promotes BDNF expression

We also verified the effect of 7,8-DHF on depressionlike behaviors in LH mice. The immobility times in the FST $\left(F_{(2,15)}=13.62\right)$ and TST $\left(F_{(2,15)}=21.96\right)$ were lower after 7,8-DHF treatment in LH mice (Fig. 8a, b, versus the LH group, $P<0.05$ ). In addition, 7,8-DHF treatment increased the BDNF and p-Akt levels in LH mice $(P<0.05$ versus the LH group, Fig. $8 \mathrm{c}-\mathrm{e}$, $\left.F_{(2,9)}=7.795\right)$. 
a

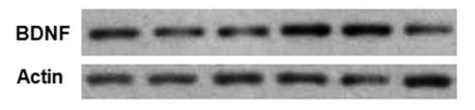

b
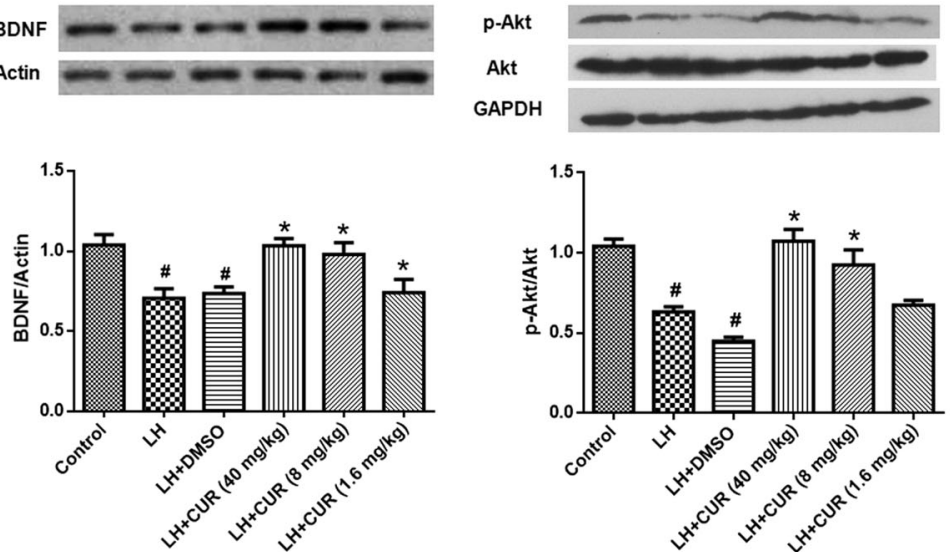

c
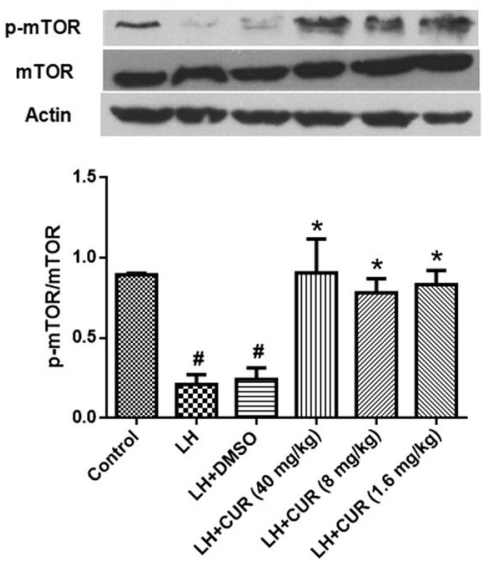

Fig. 7 Curculigoside (CUR) increases hippocampal BDNF levels and Akt-mTOR signaling in the learned helplessness (LH) model. a Upper panels are representative blots of BDNF and actin; the lower panel shows BDNF/actin quantification data. $\mathbf{b}$ Upper panels are representative blots of p-Akt, Akt, and GAPDH; the lower panel shows p-Akt/Akt quantification data. c Upper panels are representative blots of p-mTOR, mTOR, and actin; the lower panel shows quantification data of p-mTOR/mTOR. ${ }^{\#} P<0.05$ versus control, ${ }^{*} P<0.05$ versus LH
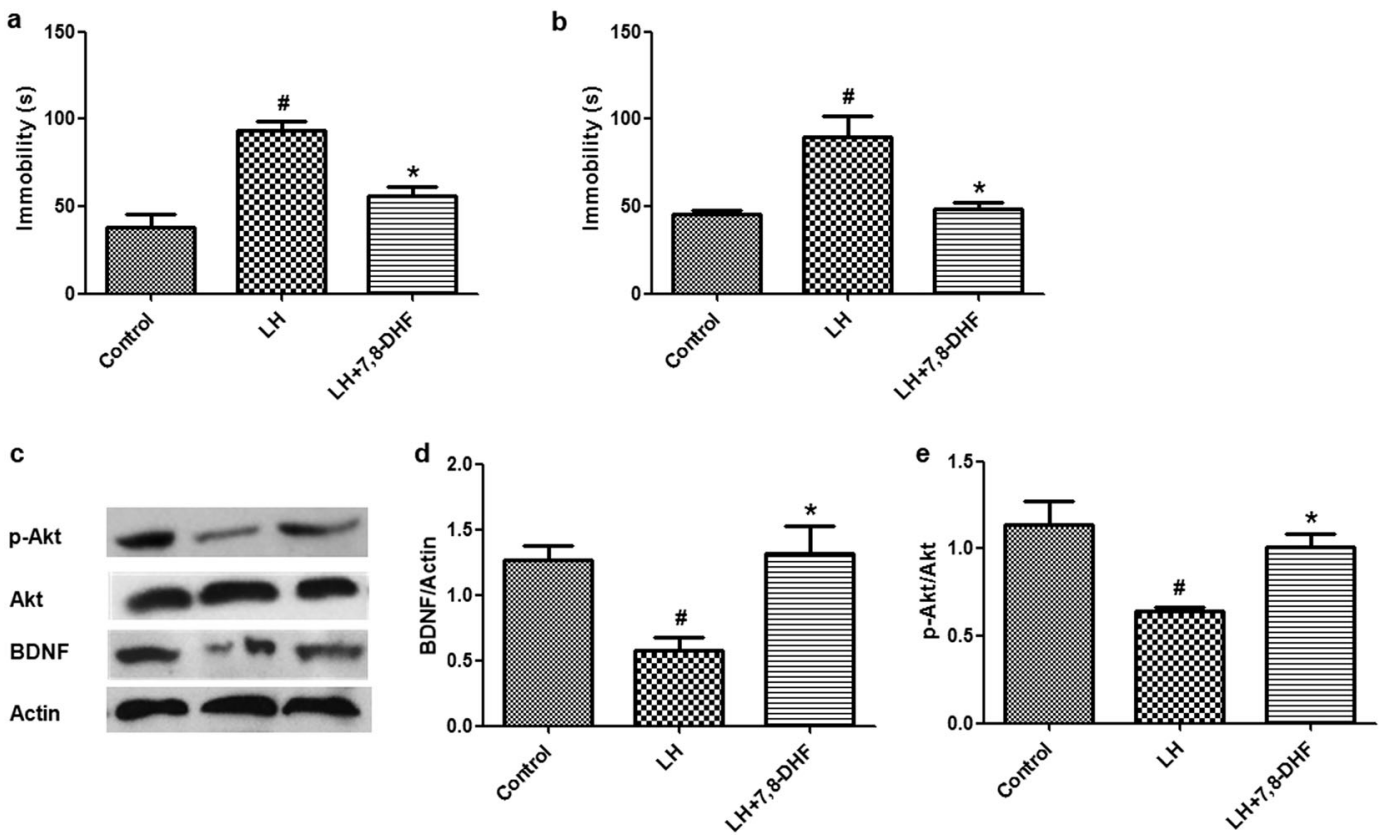

Fig. 8 7,8-DHF ameliorates depression-like behaviors in learned helplessness (LH) mice and promotes brain-derived neurotrophic factor (BDNF) expression. a Immobility time in the forced swim test. $\mathbf{b}$ Immobility time in the tail suspension test. c Representative blots of p-Akt, Akt, actin, and BDNF; d BDNF/Actin quantification data; e p-Akt/Akt quantification data. ${ }^{\#} P<0.05$ versus control, ${ }^{*} P<0.05$ versus LH

\section{DISCUSSION}

Researchers are seeking more effective antidepressants [36]. Here, we demonstrated that CUR, an active ingredient of Rhizoma Curculiginis, attenuated depression-like behaviors. Moreover, CUR facilitated fear memory extinction, promoted BDNF expression, and activated the AKT-mTOR signaling pathway. These data suggest that CUR could be a potent antidepressant in traumatic fear-induced mental disorders, such as PTSD.

The conditional fear model is widely used in the investigation of mechanisms of different processes of learning and memory. Fearrelated psychiatric disorders (e.g., phobia, PTSD) are characterized by impaired memory loss or overrecollection of events, leading to lasting changes in anxiety and depressive-like behavior. Epidemiological data have revealed that $48 \%-55 \%$ of PTSD patients exhibit depression-like behaviors [37]. The real difficulty in the treatment of this type of disease is the erasure of the fear formed in the brain. Therefore, fear extinction can be a useful method to treat this type of disease. In fact, exposure therapy has been applied in practice to treat PTSD. Laboratory work has also found that cotinine can promote fear extinction and reduce depressivelike behavior [38].

Our study initially demonstrated that there was no significant difference in freezing time after CUR treatment, suggesting that CUR did not influence memory retrieval or consolidation. Interestingly, we revealed that CUR accelerated fear memory extinction. After extinction, the immobility time in the FST and TST in the CUR groups was also lower than that in the untreated group, which indicated that CUR prevented depressive-like behavior. At the same time, correlation analysis of freezing time and immobility time showed that there was a positive correlation 
between fear memory extinction and depression-like behavior. In support of our data, increased extinction of fear memory has been shown to reduce depressive-like behavior in mice [38].

Previous studies have shown that BDNF is necessary for fear extinction $[32,33]$. In this study, we also demonstrated that CUR treatment increased hippocampal BDNF levels and TrkB phosphorylation and promoted fear extinction. More importantly, CUR increased the expression of $p$-Akt and p-mTOR but did not affect the expression of $p$-ERK. These results suggest that CUR promotes fear memory extinction and reduces depression-like behavior, which may be mediated by activating the Akt-mTOR signaling pathway to promote the expression of BDNF. 7,8-DHF, a naturally occurring flavone, has been reported to act as a potent and selective small-molecule agonist of TrkB. Most importantly, application of 7,8-DHF promotes a fast production of BDNF $[34,35]$. In our study, we further confirmed that 7,8-DHF promoted BDNF levels. Similar to CUR, 7,8-DHF also activated the Akt-mTOR signaling pathway. These data imply that CUR also promoted fear extinction using a mechanism similar to that triggered by 7,8-DHF. To support this hypothesis, immunohistochemical staining also revealed that $7,8-\mathrm{DHF}$ promoted mTOR phosphorylation.

Animals become anxious when they are repeatedly subjected to uncontrollable negative stimulation without an ability to escape. Even when facing controllable stimuli, these animals persistently exhibit less escape behavior. The $\mathrm{LH}$ paradigm is considered an animal model of depression [39]. There is sufficient evidence that LH animals exhibit several behavioral changes similar to human depression, including reduced exercise activity and sleep changes [40], as well as simultaneous neurobiochemical changes [41]. In our study, the depression-like activities caused by LH were confirmed with the TST and FST, and these depression-like behaviors were attenuated by CUR administration. Patients with depression experience cognitive impairment affecting their attention, working memory, and executive function [42]. Our MWM data showed that LH mice stayed in the target quadrant for less time than control animals. After CUR treatment, the time in the target quadrant increased significantly compared with that in the LH group. These results suggest that CUR can rescue learning and memory impairments caused by LH. Hippocampal BDNF expression was decreased in LH mice, which was consistent with a previous study [43]. Notably, this decrease in BDNF expression was reversed by CUR treatment. Our results suggest that enhanced BDNF expression might be involved in the neuroprotective effect of CUR. In addition, we found that 7,8-DHF prevented the depression-like activity induced by $\mathrm{LH}$, further confirming its previously reported antidepressant activity [34]. Moreover, we also verified a feedback effect of 7,8-DHF on BDNF expression [34, 35].

mTOR can integrate nutrition and proliferation signals, regulate cell growth and differentiation and is closely related to neuronal survival and plasticity [44]. More importantly, mTOR controls protein translation through the Akt pathway and participates in the key processes of long-term potentiation [45]. mTOR is a new target for the treatment of depression [46], but the specific mechanisms are unknown. The Akt-mTOR pathway helps maintain cell homeostasis by affecting growth, proliferation, survival, protein synthesis, and autophagy [47]. Decreased Akt and mTOR phosphorylation can lead to neuronal apoptosis. Li et al. revealed a neuroprotective effect of Akt/mTOR signaling activation in the setting of neuronal injury [48]. Similarly, mTOR phosphorylation is reduced in the amygdala of rats exposed to chronic stress [49]. Preconditioning with rapamycin eliminates the antidepressant effect of the drug [50]. For these reasons, mTOR is considered a novel target for the treatment of depression. Our results showed that LH caused a decrease in hippocampal p-Akt and p-mTOR levels and apoptosis in the DG, all of which were attenuated by CUR treatment. mTOR pathway activation is also required for new protein synthesis. We observed increased BDNF levels after $\mathrm{LH}$ animals were treated with CUR or 7,8-DHF. This cascade is likely regulated by mTOR activation.

CUR is extensively distributed in the brain after oral administration [15]. Therefore, the effects of CUR may be due to the actions of CUR itself. However, CUR also has many metabolites, among which the hydrolysis product is distributed in the brain at high levels and has anti-inflammatory and antioxidative effects [51]. Inflammation and oxidative stress are closely related to depressive behavior $[52,53]$. Another important way in which Chinese medicine is effective is by adjusting the intestinal flora. Moreover, intestinal flora imbalance is an important cause of depression [54,55]. This study mainly investigated the effect of CUR on depression by examining changes in the brain. Whether CUR plays a direct role or through other mechanisms remains to be further studied.

\section{CONCLUSION}

CUR increases the expression of BDNF in the hippocampus, thus accelerating fear memory extinction and improving depressionlike behavior. The mechanism may be mediated by activating the Akt-mTOR signaling pathway. CUR also improves depression-like behaviors and memory impairment caused by LH. These data suggest that CUR may be a potent antidepressant drug in PTSD patients.

\section{ACKNOWLEDGEMENTS}

This research was supported by the National Natural Science Foundation of China (81601181, 81673716), the Anhui Natural Science Foundation (1808085J15) and the National Key R\&D Program (2018YFC1204603).

\section{AUTHOR CONTRIBUTIONS}

GQZ conceived, designed the experiments and wrote the manuscript; SJY, ZJS, XCW, ZRZ and SBW performed the experiments and analyzed the data.

\section{ADDITIONAL INFORMATION}

Competing interests: The authors declare no competing interests.

\section{REFERENCES}

1. Chong SA, Mok YM, Subramaniam M. Depression: let's talk. Ann Acad Med Singap. 2017;46:121-3.

2. Abel JL, Rissman EF. Running-induced epigenetic and gene expression changes in the adolescent brain. Int J Dev Neurosci. 2013;31:382-90.

3. Miret M, Ayuso-Mateos JL, Sanchez-Moreno J, Vieta E. Depressive disorders and suicide: epidemiology, risk factors, and burden. Neurosci Biobehav Rev. 2013;37:2372-4.

4. Yang G, Wang Y, Zeng Y, Gao GF, Liang X, Zhou M. et al. Rapid health transition in China, 1990-2010: findings from the Global Burden of Disease Study 2010. Lancet. 2013:381:1987-2015.

5. Kulkarni S, Dhir A, Akula KK. Potentials of curcumin as an antidepressant. Sci World J. 2009;9:1233-41.

6. Watkinson A, Dockray GJ. Identification and characterization of N-glycosylated and phosphorylated variants of proenkephalin A-derived peptides in bovine adrenal medulla, spinal cord and ileum. Regul Pept. 1989;26:313-22.

7. Suris A, Holliday R, Adinoff B, Holder N, North CS. Facilitating fear-based memory extinction with dexamethasone: a randomized controlled trial in male veterans with combat-related PTSD. Psychiatry. 2017;80:399-410.

8. Bradley R, Greene J, Russ E, Dutra L, Westen D. A multidimensional meta-analysis of psychotherapy for PTSD. Am J Psychiatry. 2005;162:214-27.

9. Foa EB, McLean CP. The efficacy of exposure therapy for anxiety-related disorders and its underlying mechanisms: the case of OCD and PTSD. Annu Rev Clin Psychol. 2016;12:1-28.

10. Morina N, Sterr TN. Lack of evidence for the efficacy of psychotherapies for PTSD and depression in child and adolescent refugees. World Psychiatry. 2019;18:107-8.

11. Meir Drexler S, Merz CJ, Jentsch VL, Wolf OT. How stress and glucocorticoids timing-dependently affect extinction and relapse. Neurosci Biobehav Rev. 2018;98:145-53. 
12. Lan $Z$, Xie G, Wei M, Wang $P$, Chen $L$. The protective effect of epimedii folium and curculiginis rhizoma on Alzheimer's disease by the inhibitions of NF-kappaB/ MAPK pathway and NLRP3 inflammasome. Oncotarget. 2017;8:43709-20.

13. Ku SK, Kim JS, Seo YB, Kim YU, Hwang SL, Lee YC, et al. Effect of Curculigo orchioides on reflux esophagitis by suppressing proinflammatory cytokines. Am J Chin Med. 2012;40:1241-55.

14. Chen HY, Cho WC, Sze SC, Tong Y. Treatment of menopausal symptoms with Erxian decoction: a systematic review. Am J Chin Med. 2008;36:233-44.

15. Yuan TT, Xu HT, Zhao L, Lv L, He YJ, Zhang ND, et al. Pharmacokinetic and tissue distribution profile of curculigoside after oral and intravenously injection administration in rats by liquid chromatography-mass spectrometry. Fitoterapia. 2015;101:64-72.

16. Tundis R, Loizzo MR, Bonesi M, Menichini F. Potential role of natural compounds against skin aging. Curr Med Chem. 2015;22:1515-38.

17. Kang $Z$, Zhu H, Luan H, Han F, Jiang W. Curculigoside $A$ induces angiogenesis through VCAM-1/Egr-3/CREB/VEGF signaling pathway. Neuroscience. 2014; 267:232-40.

18. Lakshmi V, Pandey K, Puri A, Saxena RP, Saxena KC. Immunostimulant principles from Curculigo orchioides. J Ethnopharmacol. 2003;89:181-4.

19. Tian Z, Yu W, Liu HB, Zhang N, Li XB, Zhao MG, et al. Neuroprotective effects of curculigoside against NMDA-induced neuronal excitoxicity in vitro. Food Chem Toxicol. 2012;50:4010-5.

20. Zhang $K$, Liu J, You X, Kong $P$, Song $Y$, Cao L, et al. P2X7 as a new target for chrysophanol to treat lipopolysaccharide-induced depression in mice. Neurosci Lett. 2016;613:60-5.

21. Briz V, Hsu YT, Li Y, Lee E, Bi X, Baudry M. Calpain-2-mediated PTEN degradation contributes to BDNF-induced stimulation of dendritic protein synthesis. J Neurosci. 2013;33:4317-28

22. Bus BA, Molendijk ML, Tendolkar I, Penninx BW, Prickaerts J, Elzinga BM, et al. Chronic depression is associated with a pronounced decrease in serum brainderived neurotrophic factor over time. Mol Psychiatry. 2015;20:602-8.

23. Duclot $F$, Kabbaj M. Epigenetic mechanisms underlying the role of brain-derived neurotrophic factor in depression and response to antidepressants. J Exp Biol. 2015;218:21-31

24. Guilloux JP, Douillard-Guilloux G, Kota R, Wang X, Gardier AM, Martinowich $K$, et al. Molecular evidence for BDNF- and GABA-related dysfunctions in the amygdala of female subjects with major depression. Mol Psychiatry. 2012;17:1130-42.

25. Scholten AC, Haagsma JA, Cnossen MC, Olff M, van Beeck EF, Polinder S. Prevalence of and risk factors for anxiety and depressive disorders after traumatic brain injury: a systematic review. J Neurotrauma. 2016;33:1969-94.

26. Song Z, Chen H, Xu W, Wu S, Zhu G. Basolateral amygdala calpain is required for extinction of contextual fear-memory. Neurobiol Learn Mem. 2018;155:180-8.

27. Wu XY, Li JZ, Guo JZ, Hou BY. Ameliorative effects of curculigoside from Curculigo orchioides Gaertn on learning and memory in aged rats. Molecules. 2012;17:10108-18.

28. Kobayashi H, Iwata M, Mitani H, Yamada T, Nakagome K, Kaneko K. Valproic acid improves the tolerance for the stress in learned helplessness rats. Neurosci Res. 2012;72:355-63.

29. Steru L, Chermat R, Thierry B, Simon P. The tail suspension test: a new method for screening antidepressants in mice. Psychopharmacology. 1985;85:367-70.

30. Yang S, Li J, Han L, Zhu G. Early maternal separation promotes apoptosis in dentate gyrus and alters neurological behaviors in adolescent rats. Int J Clin Exp Pathol. 2018;10:10812-20.

31. Xu W, Cao J, Zhou Y, Wang L, Zhu G. GPR30 activation improves memory and facilitates DHPG-induced LTD in the hippocampal CA3 of middle-aged mice. Neurobiol Learn Mem. 2018;149:10-9.

32. $Y u$ H, Wang $Y$, Pattwell $S$, Jing $D$, Liu $T$, Zhang $Y$, et al. Variant BDNF Val66Met polymorphism affects extinction of conditioned aversive memory. J Neurosci. 2009;29:4056-64.

33. Kataoka T, Fuchikami M, Nojima S, Nagashima N, Araki M, Omura J, et al. Combined brain-derived neurotrophic factor with extinction training alleviate impaired fear extinction in an animal model of post-traumatic stress disorder. Genes Brain Behav. 2018: e12520. https://doi.org/10.1111/gbb.12520.

34. Zhang MW, Zhang SF, Li ZH, Han F. 7,8-Dihydroxyflavone reverses the depressive symptoms in mouse chronic mild stress. Neurosci Lett. 2016;635:33-8.
35. Wu CH, Hung $\mathrm{TH}$, Chen $\mathrm{CC}$, $\mathrm{Ke} \mathrm{CH}$, Lee $\mathrm{CY}$, Wang PY, et al. Post-injury treatment with 7,8-dihydroxyflavone, a TrkB receptor agonist, protects against experimental traumatic brain injury via PI3K/Akt signaling. PLoS One. 2014;9: e113397.

36. Kaster MP, Moretti M, Cunha MP, Rodrigues AL. Novel approaches for the management of depressive disorders. Eur J Pharm. 2016;771:236-40.

37. Elhai JD, Grubaugh AL, Kashdan TB, Frueh BC. Empirical examination of a proposed refinement to DSM-IV posttraumatic stress disorder symptom criteria using the National Comorbidity Survey Replication data. J Clin Psychiatry. 2008;69:597-602.

38. Alvarez-Ricartes N, Oliveros-Matus $P$, Mendoza C, Perez-Urrutia N, Echeverria F, larkov $A$, et al. Intranasal cotinine plus krill oil facilitates fear extinction, decreases depressive-like behavior, and increases hippocampal calcineurin A levels in mice. Mol Neurobiol. 2018;55:7949-60.

39. Ho YC, Wang S. Adult neurogenesis is reduced in the dorsal hippocampus of rats displaying learned helplessness behavior. Neuroscience. 2010;171:153-61.

40. Pryce CR, Azzinnari D, Spinelli S, Seifritz E, Tegethoff M, Meinlschmidt G. Helplessness: a systematic translational review of theory and evidence for its relevance to understanding and treating depression. Pharm Ther. 2011;132:242-67.

41. Muneoka K, Shirayama Y, Horio M, lyo M, Hashimoto K. Differential levels of brain amino acids in rat models presenting learned helplessness or non-learned helplessness. Psychopharmacology. 2013;229:63-71.

42. Darcet F, Gardier AM, Gaillard R, David DJ, Guilloux JP. Cognitive dysfunction in major depressive disorder. A translational review in animal models of the disease. Pharmaceuticals. 2016;9:E9 https://doi.org/10.3390/ph9010009.

43. Su CL, Su CW, Hsiao YH, Gean PW. Epigenetic regulation of BDNF in the learned helplessness-induced animal model of depression. J Psychiatr Res. 2016;76: 101-10.

44. Laplante M, Sabatini DM. mTOR signaling in growth control and disease. Cell. 2012;149:274-93.

45. Zhu G, Liu Y, Wang Y, Bi X, Baudry M. Different patterns of electrical activity lead to long-term potentiation by activating different intracellular pathways. J Neurosci. 2015;35:621-33.

46. Ka M, Condorelli G, Woodgett JR, Kim WY. mTOR regulates brain morphogenesis by mediating GSK3 signaling. Development. 2014;141:4076-86.

47. Heras-Sandoval D, Perez-Rojas JM, Hernandez-Damian J, Pedraza-Chaverri J. The role of $\mathrm{PI} / 3 \mathrm{~K} / \mathrm{AKT} / \mathrm{mTOR}$ pathway in the modulation of autophagy and the clearance of protein aggregates in neurodegeneration. Cell Signal. 2014; 26:2694-701.

48. Li W, Yang Y, Hu Z, Ling S, Fang M. Neuroprotective effects of DAHP and triptolide in focal cerebral ischemia via apoptosis inhibition and PI3K/Akt/mTOR pathway activation. Front Neuroanat. 2015;9:48.

49. Chandran A, lyo AH, Jernigan CS, Legutko B, Austin MC, Karolewicz B. Reduced phosphorylation of the mTOR signaling pathway components in the amygdala of rats exposed to chronic stress. Prog Neuropsychopharmacol Biol Psychiatry. 2013;40:240-5.

50. Liu XL, Luo L, Mu RH, Liu BB, Geng D, Liu Q, et al. Fluoxetine regulates mTOR signalling in a region-dependent manner in depression-like mice. Sci Rep. 2015;5:16024.

51. Wang L, He YJ, Han T, Zhao L, Lv L, He YQ, et al. Metabolites of curculigoside in rats and their antiosteoporotic activities in osteoblastic MC3T3-E1 cells. Fitoterapia. 2017;117:109-17.

52. Bailey DM, Brugniaux JV, Filipponi T, Marley CJ, Stacey B, Soria R, et al. Exaggerated systemic oxidative-inflammatory-nitrosative stress in chronic mountain sickness is associated with cognitive decline and depression. J Physiol. 2018;597:611-29.

53. Bouvier E, Brouillard F, Molet J, Claverie D, Cabungcal JH, Cresto N, et al. Nrf2dependent persistent oxidative stress results in stress-induced vulnerability to depression. Mol Psychiatry. 2017;22:1795.

54. Zheng P, Zeng B, Zhou C, Liu M, Fang Z, Xu X, et al. Gut microbiome remodeling induces depressive-like behaviors through a pathway mediated by the host's metabolism. Mol Psychiatry. 2016;21:786-96.

55. Winter G, Hart RA, Charlesworth RPG, Sharpley CF. Gut microbiome and depression: what we know and what we need to know. Rev Neurosci. 2018;29: 629-43. 